\title{
Erratum to: Effects of lanthanum carbonate and calcium carbonate on fibroblast growth factor 23 and hepcidin levels in chronic hemodialysis patients
}

\author{
Yu-Ming Chang ${ }^{1} \cdot$ Shih-Ching Tsai $^{1} \cdot$ Chih-Chung Shiao $^{1} \cdot$ Hung-Hsiang Liou ${ }^{2,5}$. \\ Chuan-Lan Yang ${ }^{3}$ Nai-Yu Tung ${ }^{4} \cdot \mathrm{Kua}^{-S u i} \mathrm{Hsu}^{3} \cdot$ I-Ling Chen $^{3} \cdot$ Mei-Chyn Liu ${ }^{5} \cdot$ \\ Jsun-Liang Kao ${ }^{1} \cdot$ Rong-Na Jhen $^{1} \cdot$ Ya-Ting Huang ${ }^{3}$
}

Published online: 23 February 2017

(C) Japanese Society of Nephrology 2017

\section{Erratum to: Clin Exp Nephrol \\ DOI 10.1007/s10157-016-1362-9}

In the original publication of this paper, Table 4 is incorrectly published. The MD of phosphate in calcium carbonate group is -1.033 rather than 1.033 .

The corrected Table is given here.

The online version of the original article can be found under doi:10.1007/s10157-016-1362-9.

Hung-Hsiang Liou

hh258527@ms23.hinet.net; ynk123.tw@yahoo.com.tw

1 Division of Nephrology, Department of Internal Medicine, Saint Mary's Hospital Luodong, Yilan, Taiwan

2 Division of Nephrology, Department of Internal Medicine, Hsin-Jen Hospital, 5th F, 387 Chong-Cheng Road,

Xinzhuang District, 242 New Taipei City, Taiwan

3 Department of Nursing, Saint Mary’s Hospital Luodong, Yilan, Taiwan

4 Nutrition Room, Saint Mary's Hospital Luodong, Yilan, Taiwan

5 Dialysis Center, Hsin-Ren Clinics, New Taipei City, Taiwan 
Table 4 Data before and after treatment by mixed model analysis

\begin{tabular}{|c|c|c|c|c|c|c|c|}
\hline \multirow{2}{*}{$\begin{array}{l}\text { Parameters (mean, } \\
95 \% \text { CI) }\end{array}$} & \multicolumn{3}{|c|}{ Lanthanum carbonate $(N=13)$} & \multicolumn{3}{|c|}{ Calcium carbonate $(N=12)$} & \multirow[t]{2}{*}{$p$ value (MD) } \\
\hline & Before & After & MD & Before & After & MD & \\
\hline Phosphate, mg/dL & 6.83 & 4.75 & $-2.077 * * *$ & 6.56 & 5.5 & $-1.033^{* *}$ & 0.007 \\
\hline Calcium, mg/dL & 9.24 & 9.40 & 0.162 & 9.58 & 10.04 & $0.467 *$ & 0.020 \\
\hline Albumin, g/dL & 4.22 & 4.27 & 0.046 & 4.06 & 4.10 & 0.042 & 0.206 \\
\hline ALP, U/L & 64.77 & 78.38 & 13.615 & 80.25 & 79.33 & -0.917 & 0.941 \\
\hline FGF23, pg/mL & 8677.54 & 4692.77 & $-3984.769 *$ & 9333.25 & 9262.92 & -70.333 & 0.116 \\
\hline $25(\mathrm{OH}) \mathrm{D}, \mathrm{ng} / \mathrm{mL}$ & 22.12 & 22.48 & 0.354 & 21.54 & 22.52 & 0.975 & 0.986 \\
\hline $1,25(\mathrm{OH})_{2} \mathrm{D}, \mathrm{pg} / \mathrm{mL}$ & 20.98 & 23.38 & 2.399 & 17.69 & 27.69 & 10.000 & 0.431 \\
\hline $\mathrm{iPTH}, \mathrm{pg} / \mathrm{mL}$ & 492.84 & 400.10 & -92.738 & 518.36 & 491.68 & -26.675 & 0.454 \\
\hline Fetuin-A, ng/mL & 732.49 & 653.93 & -78.562 & 724.78 & 749.83 & 25.050 & 0.303 \\
\hline Osteopontin, ng/mL & 127.38 & 160.56 & 33.185 & 193.14 & 115.18 & -77.958 & 0.295 \\
\hline Hepcidin, ng/mL & 75.10 & 68.05 & -7.042 & 66.05 & 68.92 & 2.870 & 0.965 \\
\hline hsCRP, mg/dL & 0.88 & 0.36 & -0.54 & 0.60 & 1.02 & 0.418 & 0.339 \\
\hline
\end{tabular}

Mixed model was used to analyze repeated measures and adjusted time and time $\times$ group interactions

$A L P$ alkaline phosphatase, $F G F 23$ fibroblast growth factor 23, 25( OH)D 25-hydroxy vitamin $\mathrm{D}, 1,25(\mathrm{OH})_{2} \mathrm{D}$ 1,25-dihydroxy vitamin $\mathrm{D}$, $i P T H$ intact parathyroid hormone, $h S C R P$ high sensitivity $\mathrm{C}$-reactive protein, $M D$ mean difference

${ }^{*} p<0.05$; ** $p<0.01 ; * * p<0.001$ for intragroup differences by pairwise comparisons 